\title{
Coupling of Jun amino-terminal kinase and Decapentaplegic signaling pathways in Drosophila morphogenesis
}

\author{
Bruno Glise and Stéphane Noselli ${ }^{1}$ \\ Centre de Biologie du Développement, Unité Mixte de Recherche (UMR)5547-Centre National de la Recherche Scientifique \\ (CNRS), 31062 Toulouse CEDEX, France
}

\begin{abstract}
Dorsal closure in Drosophila embryos involves the migration of two lateral epithelia toward the dorsal midline to establish the dorsal ectoderm. Previous work showed that this morphogenetic movement depends on the activities of a Jun amino (N)-terminal kinase kinase (JNKK) encoded by the hemipterous (hep) gene, and of a JNK encoded by basket. Hep is required for cell determination in the leading edge of migrating epithelia, by controlling specific expression of the puckered (puc) gene in these cells. During dorsal closure, decapentaplegic (dpp), a member of the transforming growth factor- $\beta$ (TGF- $\beta$ ) superfamily, is expressed in the row of cells making up the leading edge of the epithelia. Here, we show that the small GTPases Dcdc42, Drac1, and the Hep JNKK control $d p p$ expression in this migratory process. Appropriate $d p p$ and puc expression in the leading edge also depends on the inhibitory function of the puc gene. Further, our data suggest that the leading edge is the source of a JNK autocrine signal, and exclude a role of Dpp as such a ligand. Dorsal closure couples JNK and $d p p$ signaling pathways, a situation that may be conserved in vertebrate development.
\end{abstract}

[Key Words: Morphogenesis; hemipterous; TGF- $\beta$; MAPK pathway; cell signaling]

Received March 27, 1997; revised version accepted May 21, 1997.

Movement and folding of epithelia are basic processes in metazoan development that are required to establish germ layers and differentiated structures in the whole body. An important issue is to understand the regulatory pathways linking pattern formation and morphogenetic activity in these crucial events (Leptin 1995).

During Drosophila embryogenesis, two symmetrical cell sheets undergo coordinate migration from lateral to dorsal positions and fuse along the dorsal midline. This process, dorsal closure (DC), allows the embryo to be covered by epidermis on the dorsal side (Fig. 1A). In the context of a probable dorsoventral inversion in animal evolution, it has been proposed that $\mathrm{DC}$ is equivalent to yolk incorporation in vertebrates (for review, see NüblerJung and Arendt 1994). DC in Drosophila provides a good model system to study cell sheet movement. First, it is a simple and well-described process in which movement of epithelia depends strictly on cell elongation, requiring neither cell division nor cell recruitment (Young et al. 1993; Martinez Arias 1993). Additionally, extensive genetic screens have revealed several different loci in the genome that affect this process (Jürgens et al. 1984; Nüsslein-Volhard et al. 1984; Wieschaus et al. 1984), there-

${ }^{1}$ Corresponding author.

E-MAIL snos@cict.fr; FAX (33) 0561556507. fore offering a means to identify the entire cellular pathway.

In these DC mutants, the ectoderm does not migrate to cover the dorsal region and the resulting embryos die with a characteristic "dorsal open" phenotype. Based on molecular data, the DC genes can be divided into two main classes. In the first group, as expected of a process involving cell movement, are genes with important roles in cell adhesion [1(1) myospheroid; MacKrell et al. 1988], the cytoskeleton (zipper; Young et al. 1993) and cell junctions (coracle, canoe; Fehon et al. 1994; Miyamoto et al. 1995). These functions are crucial for establishing and/or maintaining cell shape and the integrity of the whole cell sheet. The second class comprises genes with nonstructural, signal-dependent regulatory functions. One of these regulatory genes is hemipterous (hep), which encodes a mitogen-activated protein kinase kinase (MAPKK) most related to vertebrate Jun amino (N)-terminal kinase kinase (JNKK; Glise et al. 1995). In the absence of hep function, specific expression of the puckered (puc) gene in cells of the leading edge is abolished, and movement does not occur. These data suggested that DC relies on JNK activation. In support of this view, recent work identified basket (bsk) as a Drosophila JNK gene (Riesgo-Escovar et al. 1996; Sluss et al. 1996). Further, Bsk is activated by Hep in vitro, and bsk and hep mutations provoke similar dorsal open phenotypes. An 


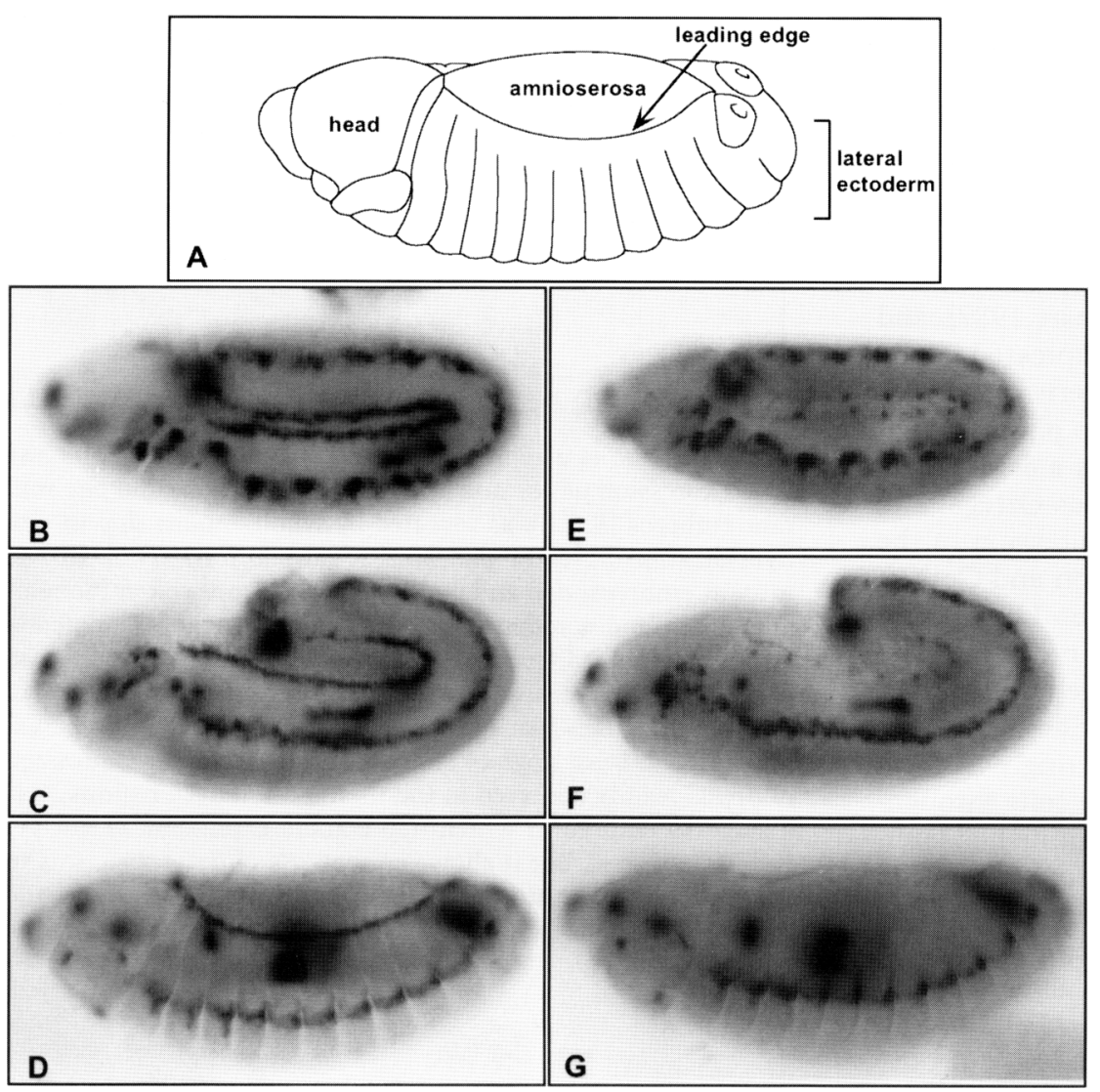

Figure 1. hep controls $d p p$ expression in the leading edge. Drawing of a stage $13 \mathrm{em}$ bryo $(A)$, showing the organization of the different structures participating in dorsal closure. During dorsal closure (stage 13-15), the leading edge and the lateral ectoderm undergo a dorsal-ward movement, resulting in the covering of the amnioserosa. On meeting at the dorsal midline, the two leading edges suture. Lateral view (adapted from Hartenstein 1993). Whole-mount $d p p$ in situ hybridizations to wild-type $(B-D)$ and hep $(E-G)$ embryos at stage $11(B, E), 12(C, F)$, and $13(D, G)$. In wild type, $d p p$ is expressed in several distinct tissues in the head and trunk regions. In the trunk ectoderm, $d p p$ is expressed as two anterior-posterior stripes, one dorsal, corresponding to the leading edge of the presumptive dorsal ectoderm, and one more ventral. In hep embryos, $d p p$ expression in the leading edge is restricted to one cell per segment during stage $11(E)$. During germ-band retraction, the "dotted" $d p p$ expression fades $(F)$. When dorsal closure begins (stage $13, D, G$ ), dpp is no longer detectable. This effect is specific to the leading edge cells, as other dpp-expressing cells remain unaffected. Anterior is left and dorsal is up. important question concerns the nature of the JNK activating signals employed in DC.

In addition to components of the Drosophila JNK pathway, other signaling molecules have a role in DC. These are members of the decapentaplegic $(d p p)$ signal transduction pathway, including schnurri, a nuclear zinc finger protein (Arora et al. 1995; Grieder et al. 1995; Staehling-Hampton et al. 1995), and the type I and type II $d p p$ receptor genes thick veins $(t k v$; Affolter et al. 1994; Brummel et al. 1994; Nellen et al. 1994; Penton et al. 1994), and punt (Letsou et al. 1995; Ruberte et al. 1995), respectively. $d p p$, a member of the transforming growth factor- $\beta$ (TGF- $\beta$ ) superfamily (Padgett et al. 1987; for review, see Kingsley 1994), is expressed in a complex pattern that reflects multiple signaling roles during Drosophila development. In early embryos, $d p p$ is established in a gradient to specify dorsal fates (Ferguson and Anderson 1992). Later, a $d p p$ signal crosses embryonic cell layers to control specific gene expression in the gut (Panganiban et al. 1990; for review, see Bienz 1994) and visceral and cardiac mesoderm (Frasch 1996). During larval development, a direct and long range Dpp morphogen activity participates in imaginal disc patterning and growth (Nellen et al. 1996), in combination with two other secreted molecules, hedgehog and wingless (for review, see Ingham 1995). Although $d p p$ mutations specifically affecting DC are unknown, genes encoding Dpp signal transducers like $t k v$ and punt have a clear zygotic dorsal open phenotype (Jürgens et al. 1984; Nüsslein-Volhard et al. 1984; Affolter et al. 1994; Letsou et al. 1995). The similar DC phenotype shown by hep and $d p p$ signal transducers therefore suggests a possible link between $d p p$ and JNK signaling in controlling dorsal closure.

In this paper, we have identified this link by showing that hep controls $d p p$ expression in the leading edge of dorsal epithelia. This identifies $d p p$ as a novel target of JNK activity and suggests a coupling of both pathways in DC. We also demonstrate that hep-mediated regulation of the two downstream targets $d p p$ and puc depends on the upstream activities of the Drosophila Drac1 and Dcdc42 genes, which encode small GTP-binding proteins with important functions in controlling cell shape and signaling (for review, see Hall 1994; Vojtek and Cooper 1995; Kyriakis and Arruch 1996; Symons 1996). We show that constitutively activated forms of Drac1 and $D c d c 42$ can mediate hep-dependent ectopic $d p p$ and $p u c$ expression in the ectoderm. These results represent the first genetic demonstration of a link between these small GTP-binding proteins and JNK signaling in a multicellular organism, and suggest a strong evolutionary conservation of the JNK pathway in vertebrates and Drosophila. Further, we show that hep and puc act antagonistically to ensure appropriate $d p p$ expression in the leading edge. Finally, experiments in which we modulate JNK activity in the leading edge suggest the existence of an autocrine JNK signal in these cells, but exclude Dpp 
as such a ligand. We discuss the role of JNK-mediated patterning in epithelial morphogenetic activity.

\section{Results}

hep controls dpp expression in the leading edge

The normal embryonic pattern of $d p p$ expression is complex and dynamic (St. Johnston and Gelbart 1987), and we focus here on those $d p p$ transcripts expressed in the dorsal ectoderm. In this region, the pattern of $d p p$ expression can be divided into two discrete phases. First, during early development (stages 5-10; for a detailed description of embryogenesis, see Campos-Ortega and Hartenstein 1985), dpp shows a broad expression in the dorsal region (data not shown; St. Johnston and Gelbart 1987), which is refined secondarily (stages 11-15), into two discrete stripes bordering this domain dorsally and ventrally (Fig. 1B-D). When dorsal closure begins (stage 13), the dorsal-most stripe is one-cell-wide and corresponds to the leading edge of the migrating ectoderm (Fig. 1D). dpp expression remains in these cells throughout DC, although expression levels are diminished later.

In hep mutant embryos, expression of $d p p$ is normal until germ band extension. At this stage, the onset of $d p p$ expression in the leading edge is affected dramatically. Instead of a continuous line of cells, only about one cell per segment expresses $d p p$ detectably (Fig. 1E). In wildtype embryos, a dotted expression is observed, but only very transiently (data not shown), indicating that the reinitiation of $d p p$ expression in the ectoderm margins is not completely synchronous. The speckled pattern of $d p p$ expression persists in hep embryos, but the signal fades during germ-band retraction (Fig. $1 F$ ). When DC begins (stage 13), dpp transcripts are no longer detected in the leading edge, in contrast to wild type (Fig. 1D,G). Targeted expression of a hep cDNA in the ectoderm completely restores $d p p$ transcripts in the leading edge (data not shown). It is noteworthy that defects in $d p p$ expression are restricted to the dorsal-most ectodermal stripe, as no change was detected in the neighboring ventral-most $d p p$ ectodermal stripe (Fig. 1G). Similar results were obtained using either of two hep alleles, hep ${ }^{1}$ or $h e p^{r 75}$ (Glise et al. 1995). These observations strongly support the specific role in DC assigned to hep (Glise et al. 1995).

Therefore, hep is required for normal $d p p$ expression in the leading edge during dorsal closure. This result suggests a role for $d p p$ in this process and identifies $d p p$ as a transcriptional target of the Hep/JNKK pathway.

\section{puc is a repressor of dpp expression in the ectoderm}

Mutations in the puc gene affect the final step of DC, which corresponds to the suture of the two lateral leading edges at the dorsal midline (Ring and Martinez Arias 1993). Expression of the puc gene, as revealed by a puclac $Z$ enhancer-trap line $\left(p u c^{E 69}\right)$, is restricted to the leading edge (Fig. 2D; Ring and Martinez Arias 1993; Glise et al. 1995|. As for $d p p$, puc expression in these cells re-

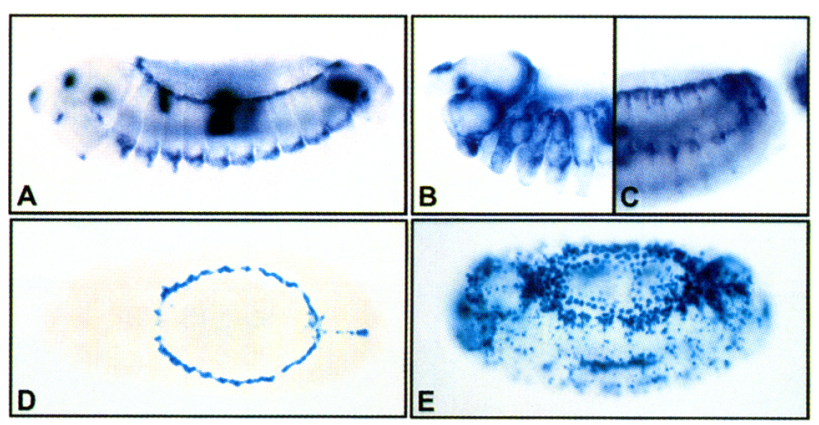

Figure 2. puc is a repressor of the JNK target genes puc and $d p p$. Expression of puc and $d p p$ in wild-type $(A, D)$ and puc mutant embryos $(B, C, E)$, as revealed by whole-mount $d p p$ in situ hybridization $(A-C)$ or puc-lacZ X-gal stainings $(D, E)$. In puc mutant embryos, $d p p$ and $p u c$ appear overexpressed in the margins $\{C, E \mid$, and ectoderm cells express both genes ectopically $(B, E)$, as compared to wild type $(A, D)$. puc-lac $Z$ staining is also observed in the amnioserosa $(E)$. Note that ectopic expression of $p u c$ and $d p p$ takes place outside the normal domain of puc expression $(D)$. $B$ is a stage 14 embryo, in which ectopic expression of $d p p$ is readily observed in the thoracic and head regions. $C$ is a stage- 13 embryo in which $d p p$ ectopic expression is not yet fully established, but it shows an increase of $d p p$ transcripts in the leading edge. Anterior is left. $D$ and $E$ are dorsal views and $A-C$ are lateral views. In $A-C$, dorsal is up. Genotypes: $(A \mid w$ embryo; $(B, C) \mathrm{puc}^{E 69} / \mathrm{puc}^{E 69} ;(D) \mathrm{puc}^{E 69} / \mathrm{TM} 3, S b ;(E) \mathrm{puc} c^{E 69} /$ puc $c^{R 10}$.

quires hep function (Glise et al. 1995). puc encodes a protein related to vertebrate dual-specificity MAPK phosphatases of the CL100 family (E. Martin-Blanco, A. Gampel, and A. Martinez Arias, pers. comm.). To test for a regulatory function of puc in the leading edge, the two target genes, $p u c$ and $d p p$, were used as in vivo markers of JNK pathway activity.

In puc mutant embryos, the levels both of $d p p$ transcripts (Fig. 2C) and of puc-lacZ activity (Fig. 2E) are increased in the leading edge compared with a wild-type situation. Intriguingly, ectopic expression of puc and $d p p$ is also induced in the ectoderm, that is, outside the normal domain of puc expression in the leading edge (Fig. $2 \mathrm{~B}, \mathrm{E})$. In addition $p u c$, but not $d p p$, is expressed ectopically in amnioserosa cells (Fig. 2E). These observations both indicate a cell non-autonomous effect of puc mutations and some intriguing features of the leading edge (see below). They also indicate that components of the hep pathway are present, but not active, in ectodermal and amnioserosa cells. puc and hep therefore appear to have antagonistic effects on $p u c$ and $d p p$ gene expression. Although hep is required specifically to activate $p u c$ and $d p p$ transcription in the leading edge (Fig. 1; Glise et al. 1995), puc functions as a repressor in the ectoderm, as evidenced by ectopic and overexpression of $p u c$ and $d p p$ in this tissue. The repressor activity of puc is hep-dependent, as in embryos mutant for hep, which also do not express puc, no ectopic expression of either puc or dpp occurs (Glise et al. 1995). This observation confirms that hep is epistatic to puc in the DC pathway. 
In summary, puc is both a repressor and a target of hep function in the leading edge. This conclusion is consistent with the molecular data indicating that puc encodes a MAPK phosphatase (E. Martin-Blanco, A. Gampel, and A. Martinez Arias, pers. comm.). The combined and antagonistic functions of hep and puc maintain appropriate levels of $p u c$ and $d p p$ activities in migrating epithelia during DC. These results indicate that a leading edge cell identity, and DC, depend on a balance between JNK activation and puc repression.

\section{Dracl and Dcdc42 are components of the Drosophila INK pathway}

To elucidate in part how hep drives specific gene expression in dorsal epithelia, we set out to identify the small GTPases involved in DC. In mammalian cells, distinct small GTPases are involved in different MAPK pathways. In particular, the vertebrate JNK and p38 pathways are regulated by the $\mathrm{Rac}$ and $\mathrm{Cdc} 42$ small GTPases (Bagrodia et al. 1995; Coso et al. 1995; Minden et al. 1995). To overcome the absence of identified mutants in the corresponding Drosophila Drac1 and Dcdc42 genes (Luo et al. 1994; Harden et al. 1995), we used the GAL4 system (Brand and Perrimon 1993) to target expression of modified Drac1 and Dcdc42 forms to specific tissues or cell types.

Activated Drac1 (Drac1V12) and Dcdc42 (Dcdc42V12; Luo et al. 1994) were expressed in populations of ectodermal cells, using GAL4 constructs expressed in the whole ectoderm $(69 B)$, in segmental stripes (en-GAL4 or $w g-G A L 4)$, or in the leading edge (LE-GAL4). In these experiments, both target genes puc and $d p p$ are induced ectopically in ectodermal cells in a pattern specific to GAL4 drivers (Fig. 3B-D; data not shown). Interestingly, we also observe an expression in amnioserosa cells, where Drac1V12 and Dcdc42V12 are not expressed (Fig. $3 \mathrm{~B}$; see below). Not all of the induced ectodermal cells show ectopic expression, indicating that some cells are refractory to activated small GTPases. Additionally, it appears that Drac1V12 and Dcdc42V12 induce distinct, though partly overlapping, responses (Fig. 3, cf. B and C). This suggests a specific effect of each, as observed in other studies (Luo et al. 1994; Eaton et al. 1995; Murphy and Montell 1996). Dcdc42V12 appears to be a good inducer of ectodermal cells located more ventrally, whereas Drac1V12 seems more active in cells nearest to the leading edge (Fig. 3B,C). The specificity of the dominant Drac1V12 and Dcdc42V12 constructs as inducers of JNK activity was tested further in experiments using activated Dras2 (Dras2V14; Brand and Perrimon 1993) or Draf, which are likely to function in two separate Ras pathways. Neither Dras2V14 nor Drafgof constructs were able to activate the JNK target genes in the ectoderm (data not shown), suggesting that Dras2 and Dras1 do not participate in the DC pathway.

Expression of activated Drac1V12 and Dcdc42V12 in the ectoderm can induce a strong activation of the two JNK target genes puc and $d p p$. These findings support the notion that the small GTPases Dracl and Dcdc42 par-

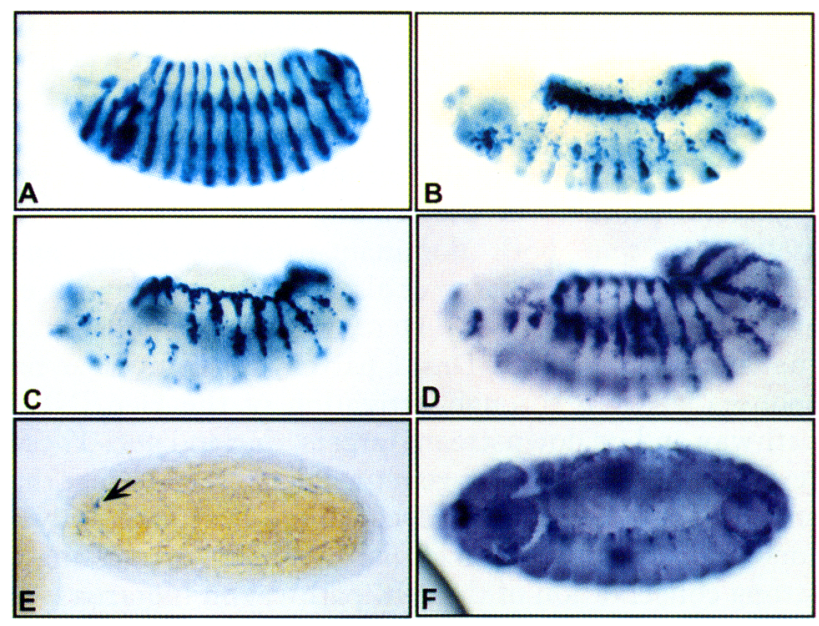

Figure 3. Drac1V12 and Dcdc42V12 induce hep-dependent ectopic puc and $d p p$ expression. Activated forms of Dracl (Drac1V12) and Dcdc42 (Dcdc42V12) were expressed using an en-GAL4 line, which is expressed in segmental stripes in the ectoderm as revealed by a UAS-lacZ reporter line $(A)$. Drac1V12 and Dcdc42V12 induce strong $d p p(D)$ and $p u c(B, C)$ expression in the ectoderm (cf. with the wild-type patterns in Fig. 2, $A$ and $D$ ), following an en pattern. Note that Drac1V12 and Dcdc42V12 induce related, but distinct responses in the ectoderm, indicating specific responsiveness of ectodermal cells to activated Drac1 and Dcdc42. In hep mutant embryos $(E, F)$, the ectopic induction of $p u c(E$, arrowhead) and $d p p(F)$ by $D c d c 42$ V12 is strongly suppressed, indicating a requirement of the downstream HEP JNKK for Dcdc42 signal transduction. Similar results were obtained with Drac1V12 (not shown). Genotypes: (A) en-GAL4/+; UAS-1acZ/+; (B) en-GAL4/+; $U A S-D r a c 1 V 12, p u c^{E 69} /+;|C|$ en-GAL4/+;UAS-Dcdc42V12, puc $^{E 69} /+;(D)$ en-GAL4/+; UAS-Dcdc42V12, puc ${ }^{E 69} /+;(E, F)$ From y $w$ hep $p^{1} / y w$ hep ${ }^{1}$; en-GAL4/en-GAL4 females crossed

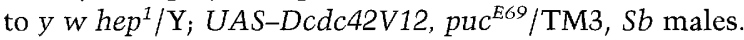

ticipate with Hep in the DC pathway and are key regulators of JNK-mediated specific gene expression.

\section{Drac1 and Dcdc42 are upstream activators of hep and dpp}

The above experiments support the notion that the small GTPases Drac1 and Dcdc42 and the Hep/JNKK participate in the same regulatory pathway, but do not allow to determine whether hep acts upstream or downstream of the GTPase activities. To determine an epistatic link among Drac1, Dcdc42 and hep, Drac1V12 and Dcdc42V12, were expressed in a hep mutant background. In these tests, hep mutations strongly suppressed the dominant effects of Drac1V12 and Dcdc42V12 (Fig. 3E,F). Only when using GAL4 lines that induce JNK activity strongly (en-GAL4;69B) was higher expression of target genes detected in some old, stage 15-17 hep mutant embryos (data not shown). We interpret this dose-dependent late activation as attributable to residual hep ${ }^{1}$ activity that had accumulated progressively to levels sufficient for target gene activation in 
these embryos. Taken together, these results lead us to place Drac1 and Dcdc42 upstream of hep in the DC pathway.

Previous reports showed that dominant-negative Drac1 affects the accumulation and rearrangement of cytoskeletal proteins in the leading edge (Harden et al. 1995) and that both dominant-negative Drac1 and Dcdc42 affect DC (Harden et al. 1995; Riesgo-Escovar et al. 1996; data not shown). Here, we have demonstrated that both $D c d c 42$ and Drac1 participate in nuclear responses as well, through activation of the Hep/JNKK pathway and its downstream targets, $d p p$ and puc. These results represent the first genetic demonstration that the small GTPases Dracl and Dcdc42 are upstream activators of JNKK in a multicellular organism, suggesting a strong conservation of the JNK pathway in metazoa (see Discussion).

\section{The leading edge is the source of a INK-activating signal}

As shown above, puc mutations have a cell nonautonomous effect, as revealed by the ectopic expression of $d p p$ and puc (Fig. 2B,E). The response is slightly different in the dorsal and ventral neighbors of the leading edge cells, because amnioserosa cells express puc only, whereas ectodermal cells express both puc and $d p p$. In puc embryos, expression in the amnioserosa precedes expression in ectodermal cells, suggesting that the amnioserosa is more sensitive to ectopic activity. Formally, these observations indicate that the leading edge cells have the potential to activate neighboring cells, and that restriction of activation to the leading edge is via puc itself. Closer observation of puc embryos indicates that ectopic expression of puc in the amnioserosa occurs preferentially in cells bordering the leading edge (Fig. 2E). Taken together, these observations suggest that the leading edge is the source of a JNK-regulated diffusible signal, whose range of activity is increased in puc mutants. To better understand how this putative signal might be controlled in the leading edge, we perturbed normal JNK activity in these cells and analyzed cell fate changes in amnioserosa and ectodermal cells, using puc as a marker. When activated Drac1V12 is expressed in the ectoderm using an en-GAL4 line, puc expression is induced in the ectoderm, as shown above (Fig. 3). Interestingly, puc expression is also detected in the amnioserosa (Fig. 3B), suggesting an inducer activity of ectodermal cells in these embryos. To test whether the leading edge, the probable site of JNK activity, is sufficient to induce puc nonautonomously in neighboring cells, activated Drac1 was expressed using a $L E-G A L 4$ line, which targets expression specifically in the leading edge (Fig. 4A,D). Expression of Drac1V12 in the leading edge specifically induces ectopic puc-lacZ in neighboring amnioserosa and ectodermal cells (Fig. 4C,F). Interestingly, this ectopic puc expression is observed predominantly in cells close to the leading edge, as noted previously in puc embryos. Using a $U A S c d c 42 V 12$ construct driven by an $L E-G A L 4$ line, ectopic induction was observed rarely, reinforcing the notion that Drac1V12 and Dcdc42V12 have different effects on JNK activity. The weaker effect observed than for puc embryos (Figs. 2 and 4, cf. E and C, respectively) presumably reflects the presence of one wild-type copy of the puc gene in these embryos. Therefore, augmenting INK activity in the leading edge, either by removing repressor functions (as in puc mutants) or by overactivation (as in activated Drac1 embryos), results in a failure of the leading edge to restrict JNK signaling.

These data provide evidence for the existence and nature of a JNK-activating signal for DC. This signal, originating from the leading edge, is diffusible and able to activate the JNK pathway in cells neighboring its source. Additionally, its range of activity depends on levels of JNK signaling, suggesting that the JNK pathway is activated, at least in part, by autocrine signaling.

\section{Dpp is not a INK-activating signal}

The conclusion that the leading edge is a local source of a JNK-activating signal is interesting, as it might reflect a general mechanism for epithelial movement (see Dis-
Figure 4. The leading edge is the source of a JNK pathway signal. $(A-C)$ Stage $14 \mathrm{em}$ bryos. $(D-F)$ are stage 15 embryos. Using a leading edge-specific $L E-G A L 4$ driver $(A$ and $D$, revealed by $U A S-l a c Z$ staining), ectopic puc can be induced in amnioserosa and ectodermal cells by Drac1V12 $(C, F)$ as compared to embryos that do not express Drac1V12 $\{B, E)$. All embryos are dorsal views, with anterior to right. To ensure high GAL4 activation in the leading edge, experiments were performed at $28^{\circ} \mathrm{C}$. UAS-lacZ produces a cytoplasmic lac $Z$ activity, whereas puc ${ }^{E 69}$ provokes nuclear stainings. Genotypes: $(A, D) L E-G A L 4$; UAS $1 a c Z /+;(B, E) U A S-D r a c 1 V 12, p u c c^{E 69} /+;(C, F)$ LE-GAL4/+; UAS-Drac1V12, puc ${ }^{E 69} /+$.

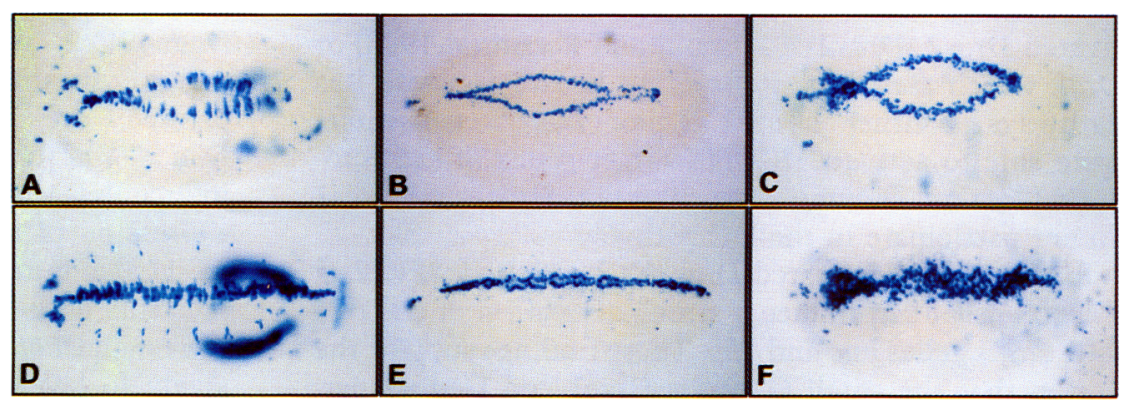


cussion). We have shown that $d p p$, which encodes a secreted molecule with signaling properties, is expressed in the leading edge in a hep-dependent manner. An attractive model is that Dpp itself could be the autocrine signal described above. To test this idea, ectopic expression of $d p p$ or of an activated $t k v$ receptor (UAS$t k v^{Q 253 D}$; Nellen et al. 1996) were induced using different GAL4 lines (en-GAL4, 69B, or $L E-G A L 4)$, and resulting $p u c$ and endogenous $d p p$ expression patterns were analyzed. Ectopic $d p p$ or $t k v^{\mathrm{Q} 253 D}$ have no effect on puc and endogenous $d p p$, in either ectoderm or amnioserosa cells (data not shown). These results therefore rule out the possibility that Dpp is sufficient to activate the JNK pathway via an autoregulatory loop. However, these observations do not exclude a paracrine signaling role for Dpp during DC, directed toward amnioserosa and/or ectodermal cells.

\section{Discussion}

Conservation of the INK pathway in invertebrates

In recent years, the study of different MAPK pathways in eukaryotes has provided a complex view of how these kinase cascades work and what they control. A current, simplified view, is that at least three related, functionally separate MAPK pathways exist in vertebrate cells: the ERK, JNK, and p38 pathways, respectively, so named for the three different MAPKs involved (Dérijeard et al. 1995; for review, see Cooper 1994; Davis 1994; Cano and Mahadevan 1995; Waskiewicz and Cooper 1995). In Drosophila, components of the ERK (for review, see Dickson and Hafen 1994; Wassarman et al. 1995; Duffy and Perrimon 1996) and JNK pathways (Glise et al. 1995; RiesgoEscovar et al. 1996; Sluss et al. 1996) have been described, and we recently isolated a Drosophila p38 kinase homolog (M. Suzanne, B. Glise, and S. Noselli, unpubl.). From these findings, we conclude that Drosophila has conserved a set of three MAPK pathways, as in vertebrates. RAS/ERK signaling in Drosophila is well conserved (Wassarman et al. 1995), and the same may be true for the Drosophila JNK pathway (Fig. 5). First, both Drosophila and vertebrate pathways are involved in the regulation of the actin cytoskeleton, cell shape (Ridley and Hall 1992; Ridley et al. 1992; Young et al. 1993; Glise et al. 1995; Harden et al. 1995; Nobes and Hall 1995), and stress responses (Coso et al. 1995; Minden et al. 1995; Bagrodia et al. 1996; Riesgo-Escovar et al. 1996; Sluss et al. 1996; for review, see Vojtek and Cooper 1995). In addition, the molecular cascade leading to JNK activation involves homologous molecules in both systems. Previous studies have demonstrated the role of the Drosophila JNKK (Hep), JNK (Bsk) and dual-specificity MAPK phosphatase (Puc) in DC (Ring and Martinez Arias 1993; Glise et al. 1995; Riesgo-Escovar et al. 1996; Sluss et al. 1996; E. Martin-Blanco, A. Gampel, and A. Martinez Arias, pers. comm.). Here, we demonstrate that the small GTPases Dracl and Dedc42 act upstream of the Hep JNKK to control gene expression, a situation well established in vertebrate cells /Coso et al. 1995;

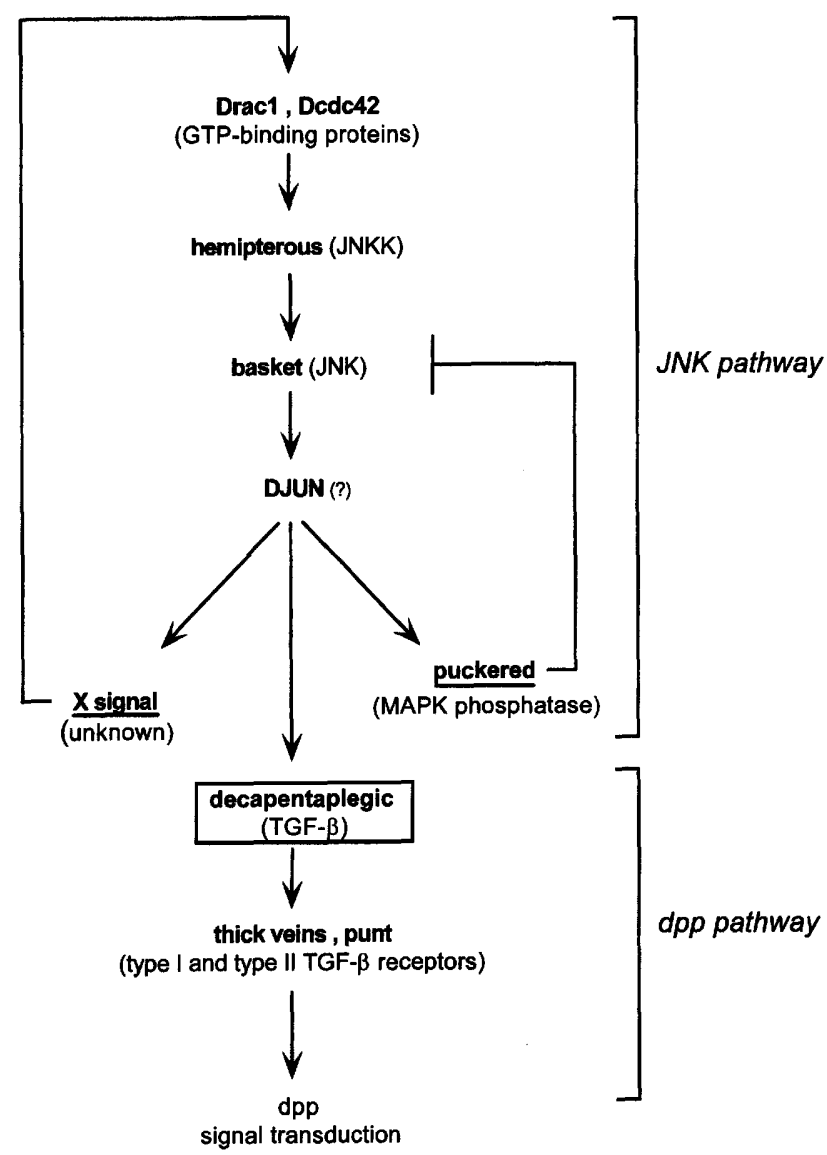

Figure 5. JNK and Dpp pathway coupling in the leading edge during dorsal closure. A putative autocrine JNK signal $(\mathrm{X})$ is synthesized in the leading edge. Following activation of leading edge cells by this signal, a protein cascade comprising the small GTPases Dracl and Dcdc42, the Hep JNKK and the Bsk JNK gene products leads to at least three different responses: maintainance of an autocrine (X) signal, and activation of the puc and $d p p$ genes in the leading edge, specifically. One candidate for mediating JNK activity in the nucleus is the Drosophila Jun homolog, DJun. puc, which encodes a Drosophila homolog of the vertebrate CL100 dual specificity phosphatases (E. MartinBlanco, A. Gampel, and A. Martinez Arias, pers. comm.), probably controls levels of JNK activity through dephosphorylation. This control is crucial in late dorsal closure, as revealed by the suture phenotype displayed by puc embryos (Ring and Martinez Arias 1993). $d p p$ is also turned on in the leading edge, and Dpp could signal (presumably by activating its Tkv and Punt type I and type II receptors, respectively/amnioserosa and/or ectodermal cells for proper movement of the whole epithelium.

Minden et al. 1995; Bagrodia et al. 1996). In addition, dominant-negative forms of these small GTPases induce dorsal open phenotypes when expressed generally in the ectoderm (Harden et al. 1995; Riesgo-Escovar et al. 1996). Interestingly, the recent analysis of a mouse hep homo$\log , \mathrm{MKK} 7$, indicates that it is also a target of rac in cell cultures (P.M. Holland, M. Suzanne, J.S. Campbell, S. Noselli, and J.A. Cooper, unpubl.). Whether Dcdc42 and Dracl induce cytoskeletal and nuclear responses independently, as shown recently for vertebrate Rac and Cdc42 (Lamarche et al. 1996), must be characterized fur- 
ther in Drosophila. A novel mediator of rac and cdc42 signaling, p65 ${ }^{\text {PAK }}$, was identified in vertebrate brain cells (Manser et al. 1994). Activated rac and cdc42 bind to the PAK serine/threonine kinase, which is required to mediate JNK activation and nuclear responses. Recently, a Drosophila PAK homolog was isolated, and shown to be accumulated in cells of the leading edge during DC (Harden et al. 1996). These data are consistent with a role for PAK as a component of the Drosophila JNK pathway. Taken together, all present data indicate a high degree of conservation of JNK pathways in vertebrate and insect cells.

\section{Coupling of INK and Dpp pathways in Drosophila development}

During Drosophila development, a complex genetic control sets the $d p p$ expression pattern. The importance of such control is illustrated by the dramatic effects on development of perturbing $d p p$ expression, either spatially (e.g., Capdevilla and Guerrero 1994; Staehling-Hampton and Hoffmann 1994; Zecca et al. 1995) or quantitatively (Morimura et al. 1996). In early embryos, $d p p$ expression along the dorsoventral axis is controlled by a dorsal protein gradient of maternal origin (for review, see Chasan and Anderson 1993). Later in embryogenesis, $d p p$ expression in the visceral mesoderm is under the direct control of the homeotic protein Ultrabithorax /Capovilla et al. 1994). During wing imaginal disc development, expression of $d p p$ along the anteroposterior boundary depends on a cascade involving the segment polarity genes engrailed and hedgehog (Zecca et al. 1995; for review, see Ingham 1995). All these data indicate that the $d p p$ gene can respond to several different developmental pathways in multiple cell types. In this paper, we identify the JNK signaling pathway as a novel key regulator of $d p p$ expression in a migratory process.

During DC, $d p p$ expression is affected dramatically in the leading edge of dorsal ectoderm, in hep embryos. $d p p$ expression in the leading edge is blocked at its onset when a dotted, rather than uniform, expression pattern is detected. This suggests that hep affects propagation and/ or maintenance of $d p p$ in these cells, rather than triggering it. However, in experiments using dominant activated Dracl and Dcdc42, we show that JNK signaling can trigger new $d p p$ expression in the ectoderm as well. These loss- and gain-of-function experiments indicate a close coupling of both pathways in Drosophila. This apparent specificity is reinforced by the inability of either activated Dras2 or Draf to modify $d p p$ expression in the ectoderm. Taken together, these findings suggest that $d p p$ is a specific JNK target in Drosophila development.

The coupling between JNK and $d p p$ in DC depends on an apparently simple link: The INK pathway activates a secondary signaling cascade, via the direct control of its ligand, Dpp (Fig. 5). No JNK-mediated $d p p$ autoregulation seems to occur in this process, as revealed by inability of either $d p p$ or $t k^{Q 253 D}$ to activate the JNK target genes. Two situations are encountered in JNK and $d p p$ coupling during DC. Initially, when $d p p$ is still silent in the leading edge, JNK and $d p p$ pathways appear to function sequentially. Once $d p p$ expression is established, however, the two pathways work in parallel and cooperate. Such coupling of JNK and $d p p$ pathways suggests two nonexclusive possibilities. First, the initial, sequential activation of JNK and $d p p$ may indicate important timing requirements, that is, $d p p$ should not be activated before JNK for proper DC to occur. This hypothesis could be tested in uncoupling experiments, where $d p p$ would be activated before the JNK pathway. In another hypothesis, coupling might serve as a way to control the JNK/Dpp ratio in the leading edge. However, we were unable to detect any effect on DC resulting from the overexpression of $d p p$ in the ectoderm or leading edge (data not shown).

Another important question is whether coupling of JNK and $d p p$ is conserved in vertebrates. Recent cloning and analysis of a novel mouse MAPKKK, TAK1 (Yamaguchi et al. 1995), suggests that it is. TAK1 activity is enhanced in cells treated with TGF- $\beta$ or BMP-4, the closest vertebrate Dpp homolog. In this model, however, coupling seems to be the opposite of what we observe in Drosophila, as JNKK activity depends on TGF- $\beta$. Although TGF- $\beta$ /TAK1 coupling has yet to be confirmed in the animal, it appears to be specific to the JNK pathway (Yamaguchi et al. 1995), as it is in Drosophila. Future studies will surely allow the cooperation between JNK and TGF- $\beta$ family members during vertebrate development to be characterized further.

\section{INK patterning and migrating epithelia}

Despite its general importance in cell and developmental biology, the way an epithelium embarks on a migratory behavior is poorly understood. The study of DC in Drosophila should provide interesting clues on how pattern formation organizes migrating epithelia. Accumulated data indicate that migrating epithelia are not homogeneous structures, but rather, count at least two different cell identities: (1) a leading edge identity, as revealed by specific gene expression (puc and dpp; Ring and Martinez Arias 1993; Glise et al. 1995) and specific protein accumulation (Martinez Arias 1993; Young et al. 1993; Harden et al. 1996); (2) a non-leading edge identity. The importance of pattern formation and leading edge determination for epithelial migration can be evaluated by mutant analysis. In hep embryos, the absence of a leading edge cell identity, as revealed by the absence of specific gene expression, results in a motionless epithelium, which otherwise shows no additional defects. The determination of the leading edge is therefore crucial for the whole migrating epithelium.

In a previous study, we showed evidence for sustained Hep activity during the spreading phase of $\mathrm{DC}$, which lasts for $\sim 2 \mathrm{hr}$ (Glise et al. 1995). Here, our data support the important notion that, during DC, the leading edge identity could be maintained by autocrine JNK-mediated signaling. During normal development, this activity seems to be restricted to the leading edge, as shown by 
the restricted puc and dpp gene activities. Under conditions of high, abnormal JNK activity in the leading edge, like those encountered in puc or activated Drac1 embryos, JNK pathway activity expands from the margins and can be detected in neighboring cells. The inducibility of cells neighboring the leading edge suggest in turn that a limiting factor in JNK pathway activation is the spatial restriction of its signal in the leading edge. Whether normal JNK activity in the leading edge has the potential to signal to other cells remains to be established. However, JNK-dependent production of Dpp, a signaling molecule that can mediate long-range morphogenic activities (Nellen et al. 1996), suggests that the leading edge could have an organizing role for the entire migrating epithelium. Determining the developmental role of $d p p$ in DC will be a future goal toward an understanding of how TGF- $\beta$ signaling can organize cell movement in epithelia. Whether the mechanisms employed in DC, that is, patterning, epithelium boundary determination, and cell signaling are conserved features of migrating tissues in other metazoa will be an exciting issue to address.

\section{Materials and methods}

\section{Genetics and GAL4 targeted expression}

A description of genetic markers and balancer chromosomes can be found in Lindsley and Zimm (1992). The puc ${ }^{E 69}$ and $p u c^{R 10}$ alleles were kindly provided by A. Martinez Arias (Ring and Martinez Arias 1993|. Homozygous germ-line clones for the hep ${ }^{r 75}$ mutation were induced by mitotic recombination as described (Glise et al. 1995).

Targeted expression of UAS-driven transgenes (Brand and Perrimon 1993) was induced using the following GAL4 lines: $69 \mathrm{~B}$ (ectodermal expression; Brand and Perrimon 1993); en-GAL4 (engrailed-like striped expression in the ectoderm); wg-GAL4 (wingless-like striped expression in the ectoderm); LE-GAL4 (leading edge specific expression; kindly provided by E. Martin Blanco and A. Martinez Arias, Cambridge University, UK). The UAS lines used in this study are: UAS-Drac1V12; UASDcdc42V12 (kindly provided by L. Luo and Y.N. Jan; Luo et al. 1994); UAS-Dras2V14 (Brand and Perrimon 1993); UAS-Drafof (a gift of N. Perrimon, Harvard University, Cambridge, MA); UAS-dpp and UAS-tkv ${ }^{\mathrm{Q} 253 D}$ (Nellen et al. 1996). puc expression was monitored using the puc-lacZ enhancer-trap line $p u c^{E 69}\left[\mathrm{p}\left(r y^{+} l a c Z\right)^{\mathrm{E} 69}\right.$; Ring and Martinez Arias 1993]. The puclac $Z$ transgene was recombined on to either GAL4 or UAS chromosomes for convenience.

For targeted expression in a hep mutant background, the following strains were constructed and used: y $w$ hep $1 / \mathrm{FM}^{1}$; enGAL4/en-GAL4; y w hep 1 /FM6; wg-GAL4/wg-GAL4; y w hep ${ }^{1} / \mathrm{FM} 6$; UAS-Drac1V12, puc E69/TM3, Sb; y w hep 1 /FM6; $U A S-D c d c 42 V 12, p u c^{E 69} / \mathrm{TM} 3, S b$. To test $d p p$ and puc expression in a hep mutant background, the following genetic crosses were made: $y w h e p^{1} / y$ w hep ${ }^{1}$; X-GAL4 females were crossed to y w hep $1 / Y$; UAS-Z, puc ${ }^{E G 9} / \mathrm{TM} 3, S b$ males. X represents any GAL4 promoter or pattern element, whereas $Z$ represents any of the UAS-driven reporter genes described above. The progeny of these crosses was collected and examined either for $d p p$ mRNA by in situ hybridization or puc ${ }^{E 69}$ lacZ expression by X-Gal stainings.

\section{Histology}

In situ hybridizations were performed according to Tautz and Pfeifle (1989), using a $d p p$ DNA probe encompassing the entire coding region (clone BEh1; St. Johnston et al. 1990). For X-Gal stainings, embryos were collected and stained for $\beta$-galactosidase activity according to standard protocols (Ashburner 1989). Photographs were taken under a Zeiss Axiophot microscope, and figures were assembled using Adobe Photoshop 3.0 software.

\section{Acknowledgments}

We thank M. Affolter, K. Basler, M. Frasch, Y.N. Jan, L. Luo, E. Martin Blanco, A. Martinez Arias, N. Perrimon, and the Bloomington Stock Center for fly strains and materials. We thank J.A. Cooper, P. Holland, E. Martin Blanco, and A. Martinez Arias for communicating unpublished results. We also thank J. Smith, A. Vincent, and all members of D. Cribbs' lab for critically reading the manuscript. We thank D. Cribbs warmly for his continuous support. B.G. is supported by the Association pour la Recherche contre le Cancer (ARC). This work is supported by the CNRS, ARC (grant 1184) and Ministère de l'Education Nationale (ACC).

The publication costs of this article were defrayed in part by payment of page charges. This article must therefore be hereby marked "advertisement" in accordance with 18 USC section 1734 solely to indicate this fact.

\section{References}

Affolter, M., D. Nellen, U. Nussbaumer, and K. Basler. 1994. Multiple requirements for the receptor serine/threonine kinase thick veins reveal novel functions of TGF $\beta$ homologs during Drosophila embryogenesis. Development 120: 31053117.

Arora, K., H. Dai, S.G. Kazuko, J. Jamal, M.B. O'Connor, A. Letsou, and R. Warrior. 1995. The Drosophila schnurri gene acts in the $d p p /$ TGF $\beta$ signaling pathway and encodes a transcription factor homologous to the human MBP family. Cell 81: 781-790.

Ashburner, M. 1989. Drosophila: A laboratory manual. Cold Spring Harbor Laboratory Press, Cold Spring Harbor, NY.

Bagrodia, S., B. Dérijard, R.J. Davis, and R.A. Cerione. 1995. Cdc42 and PAK-mediated signaling leads to Jun kinase and p38 mitogen-activated protein kinase activation. I. Biol. Chem. 270: 27995-27998.

Bienz, M. 1994. Homeotic genes and positional signalling in the Drosophila viscera. Trends Genet. 10: 22-26.

Brand, A.H. and N. Perrimon. 1993. Targeted gene expression as a means of altering cell fates and generating dominant phenotypes. Development 118: 401-415.

Brummel, T.J., V. Twombly, G. Marqués, J.L. Wrana, S.J. Newfeld, L. Attisano, J. Massagué, M.B. O'Connor, and W.M. Gelbart. 1994. Characterization and relationship of $d p p$ receptors encoded by the saxophone and thick veins genes in Drosophila. Cell 78: 251-261.

Campos-Ortega, J.A. and V. Hartenstein. 1985. The embryonic development of Drosophila melanogaster. Springer, Berlin, Germany.

Cano, E. and L.C. Mahadevan. 1995. Parallel signal processing among mammalian MAPKs. Trends Biochem. Sci. 20: 117-122.

Capdevilla, J. and I. Guerrero. 1994. Targeted expression of the signaling molecule decapentaplegic induces pattern duplications and growth alterations in Drosophila wings. EMBO $\%$ 13: 4459-4468. 
Capovilla, M., M. Brandt, and J. Botas. 1994. Direct regulation of decapentaplegic by Ultrabithorax and its role in Drosophila midgut morphogenesis. Cell 76: 461-475.

Chasan, R. and K. Anderson. 1993. Maternal control of dorsalventral polarity and pattern in the embryo. In The development of Drosophila (ed. M. Bate and A. Martinez Arias), pp. 387-424. Cold Spring Harbor Laboratory Press, Cold Spring Harbor, NY.

Cooper, J.A. 1994. Straight and narrow or tortuous and intersecting? Curr. Biol. 4: 1118-1121.

Coso, O.A., M. Chiariello, J.C. Yu, H. Teramoto, P. Crespo, N. $\mathrm{Xu}$, T. Miki, and J.S. Gutkind. 1995. The small GTP-binding proteins $\mathrm{Racl}$ and $\mathrm{Cdc} 42$ regulate the activity of the JNK/ SAPK signaling pathway. Cell 81: 1137-1146.

Davis, J.D. 1994. MAPKs: New JNK expands the group. Trends Biol. Sci. 19: 470-473.

Dérijard, B., J. Raingeaud, T. Barret, I.H. Wu, J. Han, R.J. Ulevitch, and R. Davis. 1995. Independent human MAP kinase signal transduction pathways defined by MEK and MKK isoforms. Science 267: 682-685.

Dickson, B. and E. Hafen. 1994. Genetics of signal transduction in invertebrates. Curr. Opin. Genet. Dev. 4: 64-70.

Duffy, J.B. and N. Perrimon. 1996. Recent advances in understanding signal transduction pathways in worms and flies. Curr. Opin. Cell Biol. 8: 231-238.

Eaton, S., P. Auvinen, L. Luo, Y.N. Jan, and K. Simons. 1995. CDC42 and Rac1 control different actin-dependent processes in the Drosophila wing disc epithelium. I. Cell Biol. 131: 151-164.

Fehon, R.G., I.A. Dawson, and S. Artavanis-Tsakonas. 1994. A Drosophila homologue of membrane-skeleton protein 4.1 is associated with septate junctions and is encoded by the coracle gene. Development 120: 545-557.

Ferguson, E.L. and K.V. Anderson. 1992. Decapentaplegic acts as a morphogen to organize dorsal-ventral pattern in the Drosophila embryo. Cell 71: 45I-461.

Frasch, M. 1995. Induction of visceral and cardiac mesoderm by ectodermal Dpp in the early Drosophila embryo. Nature 374: 464-467.

Glise, B., H. Bourbon, and S. Noselli. 1995. hemipterous encodes a novel Drosophila MAP kinase kinase, required for epithelial cell sheet movement. Cell 83: 451-461.

Grieder, N.C., D. Nellen, R. Burke, K. Basler, and M. Affolter. 1995. schnurri is required for Drosophila dpp signaling and encodes a zinc finger protein similar to the mammalian transcription factor PRDII-BF1. Cell 81: 791-800.

Hall, A. 1994. Small GTP-binding proteins and the regulation of the actin cytoskeleton. Annu. Rev. Cell Biol. 10: 31-54.

Harden, N., H.Y. Loh, W. Chia, and L. Lim. 1995. A dominant inhibitory version of the small GTP-binding protein Rac disrupts cytoskeletal structures and inhibits developmental cell shape changes in Drosophila. Development 121:903914.

Harden, N., J. Lee, H.Y. Loh, Y.M. Ong, I. Tan, T. Leung, E. Manser, and L. Lim. 1996. A Drosophila homolog of the Racand cdc42-activated serine/threonine kinase PAK is a potential focal adhesion and focal complex protein that colocalizes with dynamic actin structures. Mol. Cell. Biol. 16: 18961908.

Hartenstein, V. 1993. Atlas of Drosophila development. In The development of Drosophila (ed. M. Bate and A. Martinez Arias), Cold Spring Harbor Laboratory Press, Cold Spring Harbor, NY.

Ingham, P. 1995. Signalling by hedgehog family proteins in Drosophila and vertebrate development. Curr. Opin. Genet. Dev. 5: 492-498.
Jürgens, G., E. Wieschaus, C. Nüsslein-Volhard, and H. Kluding. 1984. Mutations affecting the pattern of the larval cuticle in Drosophila melanogaster II. Zygotic loci on the third chromosome. Wilhelm Roux's Arch. Dev. Biol. 193: 283295.

Kingsley, D.M. 1994. The TGF- $\beta$ superfamily: New members, new receptors, and new genetic tests of function in different organisms. Genes \& Dev. 8: 133-146.

Kyriakis, J.M. and J. Avruch. 1996. Protein kinase cascades activated by stress and inflammatory cytokines. BioEssays 18: 577.

Lamarche, N., N. Tapon, L. Stowers, P.D. Burbelo, P. Aspenström, T. Bridges, J. Chant, and A. Hall. 1996. Rac and cdc42 induce actin polymerization and Gl cell cycle progression independently of p65PAK and the JNK/SAPK MAP kinase cascade. Cell 87: 519-529.

Leptin, M. 1995. Drosophila gastrulation: From pattern formation to morphogenesis. Annu. Rev. Cell Dev. Biol. 11: 189212.

Letsou, A., A. Arora, J.L. Wrana, K. Simin, V. Twombly, J. Jamal, K. Staehling-Hampton, F.M. Hoffmann, W.M. Gelbart, J. Massagué, and M.B. O'Connor. 1995. Drosophila dpp signaling is mediated by the punt gene product: A dual ligandbinding type II receptor of the TGF $\beta$ receptor family. Cell 80: 899-908.

Lindsley, D.L. and G.G. Zimm. 1992. The genome of Drosophila melanogaster. Academic Press, San Diego, CA.

Luo, L., Y.J. Liao, L.Y. Jan, and Y.N. Jan. 1994. Distinct morphogenetic functions of similar GTPases: Drosophila Drac1 is involved in axonal outgrowth and myoblast fusion. Genes \& Dev. 8: 1787-1802.

MacKrell, A.J., B. Blumberg, S.R. Haynes, and J.H. Fessler. 1988. The lethal myospheroid gene of Drosophila encodes a membrane protein homologous to vertebrate integrin $\beta$ subunits. Proc. Nat1. Acad. Sci. 85: 2633-2637.

Manser, E., T. Leung, H. Salihuddin, Z.S. Zhao, and L. Lim. 1994. A brain serine/threonine protein kinase activated by Cdc42 and Rac1. Nature 367: 40-46.

Martinez Arias, A. 1993. Development and patterning of the larval epidermis of Drosophila. In The development of Drosophila melanogaster, Vol. I, (ed. M. Bate and A. Martinez Arias), pp. 517-608. Cold Spring Harbor Laboratory Press, Cold Spring Harbor, NY.

Minden, A., A. Lin, F.-X. Claret, A. Abo, and M. Karin. 1995. Selective activation of the JNK signaling cascade and c-Jun transcriptional activity by the small GTPases Rac and Cdc42Hs. Cell 81: 1147-1157.

Miyamoto, H., I. Nihonmatsu, S. Kondo, R. Ueda, S. Togashi, K. Hirata, Y. Ikegami, and D. Yamamoto. 1995. canoe encodes a novel protein containing a GLGF/DHR motif and functions with Notch and scabrous in common developmental pathways in Drosophila. Genes \& Dev. 9: 612-625.

Morimura, S., L. Maves, Y. Chen, and F.M. Hoffmann. 1996. decapentaplegic overexpression affects Drosophila wing and leg imaginal disc development and wingless expression. Dev. Biol. 177: 136-151.

Murphy, A.M. and D.J. Montell. 1996. Cell type-specific roles for cdc42, Rac, and RhoL in Drosophila oogenesis. I. Cell Biol. 133: 617-630.

Nellen, D., M. Affolter, and K. Basler. 1994. Receptor serine/ threonine kinases implicated in the control of Drosophila body pattern by decapentaplegic. Cell 78: 225-237.

Nellen, D., R. Burke, G. Struhl, and K. Basler. 1996. Direct and long-range action of a dpp morphogen gradient. Cell 85: $357-$ 368.

Nobes, C.D. and A. Hall. 1995. Rho, Rac, and Cdc42 GTPases 
regulate the assembly of multimolecular focal complexes associated with actin stress fibers, lamellipodia, and filipodia. Cell 81: 53-62.

Nübler-Jung, K. and D. Arendt. 1994. Is ventral in insects dorsal in vertebrates? Wilhelm Roux's Arch. Dev. Biol. 203: 357366.

Nüsslein-Volhard, C., E. Wieschaus, and H. Kluding. 1984. Mutations affecting the pattern of the larval cuticle in Drosophila melanogaster I. Zygotic loci on the second chromosome. Wilhelm Roux's Arch. Dev. Biol. 193: 267-282.

Padgett, R.W., R.D. St. Johnston, and W.M. Gelbart. 1987. A transcript from a Drosophila pattern gene predicts a protein homologous to the transforming growth factor- $\beta$ family. $\mathrm{Na}$ ture 325: 81-84.

Panganiban, G.E.F., R. Reuter, M.P. Scott, and F.M. Hoffmann. 1990. A Drosophila growth factor homolog, decapentaplegic, regulates homeotic gene expression within and accross germ layers during midgut morphogenesis. Development 110: 1041-1050.

Penton, A., Y. Chen, K. Staehling-Hampton, J.L. Wrana, L. Attisano, J. Szidonya, J.A. Cassill, J. Massagué, and F.M. Hoffmann. 1994. Identification of two bone morphogenetic protein type I receptors in Drosophila and evidence that Brk25D is a decapentaplegic receptor. Cell 78: 239-250.

Ridley, A.J. and A. Hall. 1992. The small GTP-binding protein rho regulates the assembly of focal adhesions and actin stress fibers in response to growth factors. Cell 70: 389-399.

Ridley, A.J., H.F. Paterson, C.L. Johnston, D. Diekmann, and A. Hall. 1992. The small GTP-binding protein rac regulates growth factor-induced membrane ruffling. Cell 70:401410.

Riesgo-Escovar, J.R., M. Jenni, A. Fritz, and E. Hafen. 1996. The Drosophila Jun-N-terminal kinase is required for cell morphogenesis but not for DJun-dependent cell fate specification in the eye. Genes \& Dev. 10: 2759-2768.

Ring, J.M. and A. Martinez Arias. 1993. puckered, a gene involved in position-specific cell differentiation in the dorsal epidermis of the Drosophila larva. Development (Suppl.) 251-259.

Ruberte, E., T. Marty, D. Nellen, M. Affolter, and K. Basler. 1995. An absolute requirement for both the type II and type I receptors, punt and thick veins, for $d p p$ signaling in vivo. Cell 80: 889-897.

Sluss, H.K., Z. Han, T. Barret, R.J. Davis, and Y.T. Ip. 1996. A INK signal transduction pathway that mediates morphogenesis and immune response in Drosophila. Genes \& Dev. 10: $2745-2758$.

St. Johnston, R.D. and W.M. Gelbart. 1987. Decapentaplegic transcripts are localized along the dorsal-ventral axis of the Drosophila embryo. EMBO I. 6: 2785-2791.

St. Johnston, R.D., F.M. Hoffmann, R.K. Blackman, D. Segal, R. Grimaila, R.W. Padgett, H.A. Irick, and W.M. Gelbart. 1990. Molecular organization of the decapentaplegic gene in Drosophila melanogaster. Genes \& Dev. 4: 1114-1127.

Staehling-Hampton, K. and F.M. Hoffmann. 1994. Ectopic decapentaplegic in the Drosophila midgut alters the expression of five homeotic genes, $d p p$, and wingless, causing specific morphogenetic defects. Dev. Biol. 164: 502-512.

Staehling-Hampton, K., A.S. Laughon, and F.M. Hoffmann. 1995. A Drosophila protein related to the human zinc finger transcription factor PRDI/MBPI/HIV-EP1 is required for dpp signaling. Development 121: 3393-3403.

Symons, M. 1996. Rho family GTPases: The cytoskeleton and beyond. Trends Biol. Sci. 21: 178-181.

Tautz, D. and C. Pfeifle. 1989. A non-radioactive in situ hybridization method for the localization of specific RNAs in Dro- sophila embryos reveals translational control of the segmentation gene hunchback. Chromosoma 98: 81-85.

Voitek, A.B. and J.A. Cooper. 1995. Rho family members: Activators of MAP kinase cascades. Cell 82: 527-529.

Waskiewicz, A.J. and J.A. Cooper. 1995. Mitogen and stress response pathways: MAP kinase cascades and phosphatase regulation in mammals and yeast. Curr. Opin. Cell Biol. 7: 798-805.

Wassarman, D.A., M. Therrien, and G.M. Rubin. 1995. The Ras signaling pathway in Drosophila. Curr. Opin. Genet. Dev. 5: 44-50.

Wieschaus, E., C. Nüsslein-Volhard, and G. Jürgens. 1984. Mutations affecting the pattern of the larval cuticle in Drosophila melanogaster III. Zygotic loci on the X-chromosome and the fourth chromosome. Wilhelm Roux's Arch. Dev. Biol. 193: 296-307.

Yamaguchi, K., K. Shirakabe, H. Shibuya, K. Irie, I. Oishi, N. Ueno, T. Taniguchi, E. Nishida, and K. Matsumoto. 1995. Identification of a member of the MAPKKK family as a potential mediator of TGF- $\beta$ signal transduction. Science 270: 2008-2011.

Young, P.E., A.M. Richman, A.S. Ketchum, and D.P. Kiehart. 1993. Morphogenesis in Drosophila requires nonmuscle myosin heavy chain function. Genes \& Dev. 7: 29-41.

Zecca, M., K. Basler, and G. Struhl. 1995. Sequential organizing activities of engrailed, hedgehog and decapentaplegic in the Drosophila wing. Development 121: 2265-2278. 


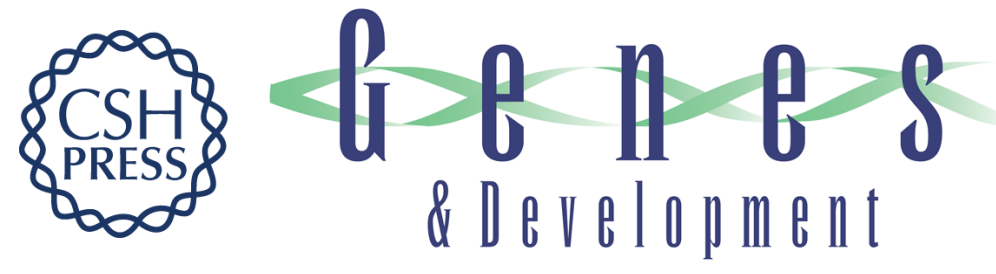

\section{Coupling of Jun amino-terminal kinase and Decapentaplegic signaling pathways in Drosophila morphogenesis.}

B Glise and S Noselli

Genes Dev. 1997, 11:

Access the most recent version at doi:10.1101/gad.11.13.1738

References This article cites 64 articles, 21 of which can be accessed free at:

http://genesdev.cshlp.org/content/11/13/1738.full.html\#ref-list-1

License

Email Alerting

Service

Receive free email alerts when new articles cite this article - sign up in the box at the top right corner of the article or click here.

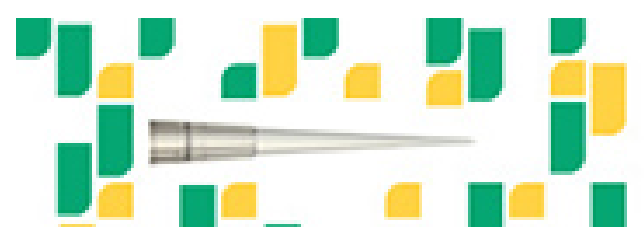

Focused on your science.

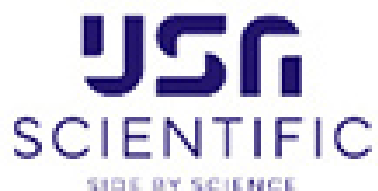

Copyright (c) Cold Spring Harbor Laboratory Press 